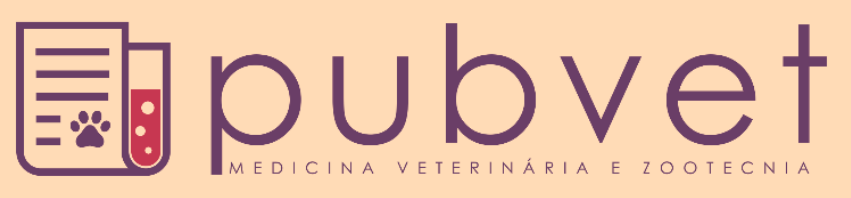

https://doi.org/10.31533/pubvet.v15n01a723.1-6

\title{
Doxorrubicina no tratamento de adenocarcinoma de glândula salivar
}

\author{
Felipe Noleto de Paiva ${ }^{1 * \bullet}$ (D), Lizandra de Fátima Brandão Torquato ${ }^{2}$, Gabriella Santos \\ Oliveira $^{2} \bullet$, Shayne Abranches de Souza ${ }^{2} \bullet$ (D), Dayane Caicó Collares Araujo ${ }^{\circ}{ }^{\circ}$, Thiago Souza \\ $\operatorname{Costa}^{3}{ }^{\circ}$, Julio Israel Fernandes ${ }^{\circ} \odot$
}

${ }^{I}$ Médico Veterinário residente, Universidade Federal Rural do Rio de Janeiro, Rio de Janeiro - RJ, Brasil.

${ }^{2}$ Graduando em Medicina Veterinária, Universidade Federal Rural do Rio de Janeiro, Rio de Janeiro- RJ, Brasil

${ }^{3}$ Médico Veterinário doutorando, Universidade Federal Rural do Rio de Janeiro, Rio de Janeiro - RJ, Brasil

${ }^{4}$ Professor Associado, Universidade Federal Rural do Rio de Janeiro, Departamento de Medicina e Cirurgia Veterinária-RJ, Brasil.

*Autor para contato: n-paiva@hotmail.com

Resumo. Neoplasias que acometem as glândulas salivares de cães e gatos são extremamente raras, não sendo reportadas predileção por raça ou sexo. Os sinais clínicos incluem o aumento de volume local como achado mais frequente, e manifestações em consequência desse aumento. $O$ diagnóstico definitivo se dá através do exame histopatológico, porém a citologia aspirativa por agulha fina se mostra um bom exame de triagem diagnóstica. Exames de imagem como radiografia, ultrassonografia e tomografia computadorizada podem auxiliar no estadiamento e planejamento terapêutico. O tratamento mais indicado é a ressecção cirúrgica total, que nem sempre é viável, sendo nestes casos a radioterapia a terapia preconizada. Estudos com quimioterapia são muito raros nesse tipo de afecção neoplásica. O objetivo do relato foi reportar o caso de um canino de treze anos de idade, com presença de massa irregular, firme, parcialmente aderida, medindo aproximadamente $8,5 \times 7,6 \times 4,0 \mathrm{~cm}$, em região submandibular e evolução de 4 meses, já com diagnóstico histopatológico de carcinoma de glândula salivar após biopsia excisional. Foram realizados exames de ultrassonografia cervical, abdominal e radiografia torácica para investigação geral e avaliação da viabilidade cirúrgica. Nova cirurgia foi realizada, porém sem informações acerca de margens de segurança por exame histopatológico, sendo preconizada a quimioterapia adjuvante com a combinação da doxorrubicina e ciclofosfamida. $\mathrm{O}$ animal veio a óbito após quatro sessões manifestando sinais de recidiva tumoral. Dados em literatura relacionados ao tratamento quimioterápico para neoplasias de glândulas salivares ainda são escassos e novos estudos são necessários.

Palavras-chave: Adenocarcinoma, canino, neoplasia, quimioterapia

\section{Doxorubicin in the treatment of salivary gland adenocarcinoma}

Abstract. Neoplasms that affect the salivary glands of dogs and cats are extremely rare, with no predilection for race or sex being reported. Clinical signs include an increase in local volume as the most frequent finding, and manifestations as a result of this increase. The definitive diagnosis is made through histopathological examination, but fine needle aspiration cytology is a good diagnostic screening test. Imaging tests such as radiography, ultrasound and computed tomography can assist in staging and therapeutic planning. The most suitable treatment is total surgical resection, which is not always feasible, in which case radiotherapy is the recommended therapy. Chemotherapy studies are very rare in this type of neoplastic disease. The objective of the report was to report the case of a thirteenyear-old canine, with the presence of irregular, firm, partially adherent mass, measuring approximately $8.5 \times 7.6 \times 4.0 \mathrm{~cm}$, in the submandibular region and evolution of 4 months, already with histopathological diagnosis of salivary gland carcinoma after excisional 
biopsy. Cervical, abdominal and chest X-ray examinations were performed for general investigation and evaluation of surgical viability. A new surgery was performed, but without information about safety margins due to histopathological examination, and adjuvant chemotherapy with the combination of doxorubicin and cyclophosphamide was recommended. The animal died after four sessions showing signs of tumor recurrence. Literature data related to chemotherapy treatment for salivary gland neoplasms are still scarce and further studies are needed.

Keywords: Adenocarcinoma, canine, neoplasia, chemotherapy

\title{
Doxorrubicina en el tratamiento del adenocarcinoma de glándulas salivales
}

\begin{abstract}
Resumen. Las neoplasias que afectan las glándulas salivales de perros y gatos son extremadamente raras, sin que se reporten predilecciones por raza o sexo. Los signos clínicos incluyen un aumento en el volumen local como el hallazgo más frecuente y manifestaciones como resultado de este aumento. El diagnóstico definitivo se realiza a través del examen histopatológico, pero la citología por aspiración con aguja fina es una buena prueba diagnóstica de detección. Las pruebas de imagen como la radiografía, el ultrasonido y la tomografía computarizada pueden ayudar en la estadificación y la planificación terapéutica. El tratamiento más adecuado es la resección quirúrgica total, que no siempre es factible, en cuyo caso la radioterapia es la terapia recomendada. Los estudios de quimioterapia son muy raros en este tipo de enfermedad neoplásica. El objetivo del informe fue informar el caso de un canino de trece años, con presencia de masa irregular, firme y parcialmente adherente, que medía aproximadamente $8.5 \times 7.6 \times 4.0 \mathrm{~cm}$, en la región submandibular y evolución de 4 meses, ya con diagnóstico histopatológico de carcinoma de glándulas salivales después de una biopsia por escisión. Se realizaron exámenes de ultrasonido cervical y abdominal y radiografía de tórax para la investigación general y la evaluación de la viabilidad quirúrgica. Se realizó una nueva cirugía, pero sin información sobre los márgenes de seguridad debido al examen histopatológico, y se recomendó quimioterapia adyuvante con la combinación de doxorrubicina y ciclofosfamida. El animal murió después de cuatro sesiones mostrando signos de recurrencia tumoral. Los datos de la literatura relacionados con el tratamiento de quimioterapia para las neoplasias de las glándulas salivales aún son escasos y se necesitan más estudios.
\end{abstract}

Palabras clave: Adenocarcinoma, canino, neoplasia, quimioterapia

\section{Introdução}

Entre as afecções das glândulas salivares, as neoplasias representam cerca de $30 \%$ dos casos, configurando como uma causa significativa de lesão nesse tecido, tendo como principal diagnóstico diferencial as mucoceles (Hammer et al., 2001; Spangler \& Culbertson, 1991). No entanto, em relação ao total de tumores, essas neoplasias são raras em cães e gatos, compreendendo $0,17 \%$ dos casos (Carberry, 1988). Não há predileção sexual ou racial descrita, e a etiologia não é completamente elucidada, havendo participação de fatores ambientais e intrínsecos do animal (Collins et al., 2000; Rosenthal, 2004). Em relação aos subtipos histológicos, os adenocarcinomas são observados com maior frequência (Head, 2003; Meuten, 2016; Sozmen et al., 2003).

Os sinais clínicos são inespecíficos e geralmente incluem halitose, disfagia, exoftalmia e inchaço unilateral, firme e indolor, podendo se localizar na região superior do pescoço (glândulas mandibular e sublingual), base da orelha (glândula parótida), lábio superior ou maxila (glândula zigomática) e membrana mucosa do lábio e língua (tecido salivar acessório) (Collins et al., 2000; Rosenthal, 2004). O comportamento tende a cursar com invasão local, podendo realizar metástases rapidamente para linfonodos regionais (Hammer et al., 2001).

A citologia aspirativa por agulha fina (CAAF) representa um exame de triagem e auxilia na suspeita diagnóstica e na diferenciação dos casos de mucocele e outros diagnósticos diferencias (Meuten, 2016). No entanto, somente pelo exame histopatológico o diagnóstico definitivo é obtido. Exames de imagem 
de radiografia torácica, ultrassonografia (US) abdominal e cervical e tomografia computadorizada (TC) de cabeça podem auxiliar no estadiamento e planejamento terapêutico (Withrow et al., 2020).

O tratamento mais indicado é a ressecção cirúrgica, porém nem sempre é possível, devido ao comprometimento de estruturas vitais adjacentes. Nestes casos, a radioterapia é a modalidade indicada (Hammer et al., 2001). A quimioterapia não tem seus efeitos comprovados, devido à escassez de informações na literatura, mas pode ser indicada em caso de metástases, histopatologia altamente maligna (Withrow et al., 2020) e em casos de margem cirúrgica comprometida (Hammer et al., 2001).

\section{Relato de caso}

Um canino, fêmea, castrada, sem raça definida (SRD), de treze anos de idade foi atendido no Serviço de Oncologia do Hospital Veterinário (HV) da Universidade Federal Rural do Rio de Janeiro (UFRRJ), manifestando aumento de volume em região cervical, com evolução de quatro meses. Segundo o tutor, o primeiro atendimento havia se dado em veterinário externo, onde foi realizada a exérese cirúrgica do nódulo e envio do material para avaliação histopatológica. Em laudo externo foi descrito presença de células epiteliais neoplásicas cuboides, dispostas em túbulos de lúmen preenchido por conteúdo secretório anfofílico, com diagnóstico de carcinoma de glândula salivar. Após a cirurgia, foi observado o surgimento de pequenos nódulos no local apresentando crescimento gradual.

No momento da consulta o animal apresentava-se em bom estado geral. Durante a anamnese, constatou-se normorexia, normoquesia, normodipsia e normoúria. No exame físico, os parâmetros vitais apresentavam-se dentro dos valores de normalidade para a espécie. Em região submandibular esquerda, notava-se massa irregular, firme, parcialmente aderida, medindo aproximadamente $8,5 \times 7,6 \times 4,0 \mathrm{~cm}$ (Figura 1). Linfonodos não reativos. Foi realizada citologia da lesão, com resultado compatível com neoplasia epitelial glandular (Figura 2), com evidência de critérios de malignidade de anisocitose, anisocariose, relação núcleo:citoplasma aumentada, dentre outros, sendo compatível com o diagnóstico prévio.

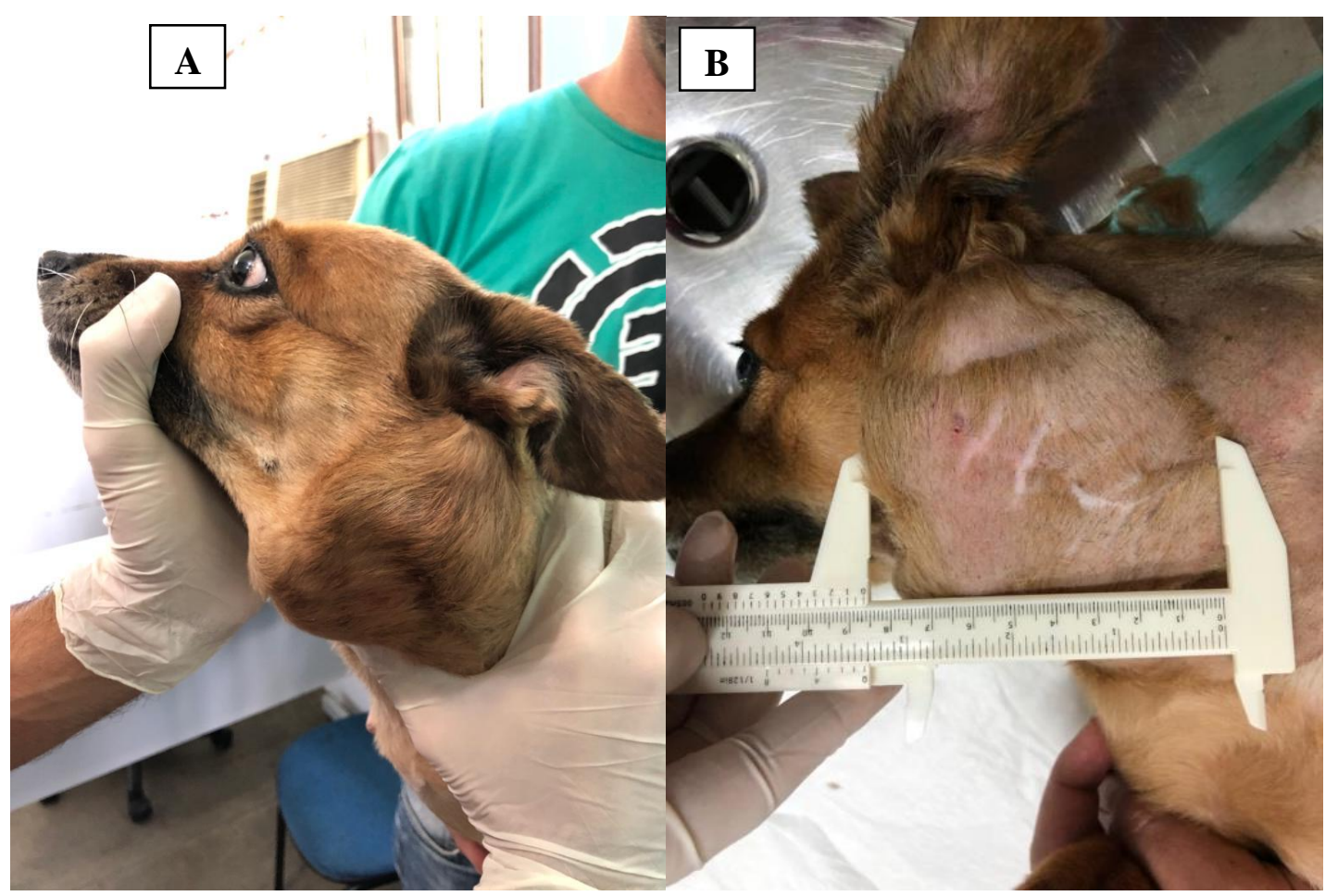

Figura 1. Canino manifestando massa de aproximadamente 8,5 x 7,6 x 4,0 cm em região submandibular esquerda. Aspecto macroscópico das lesões. A: vista lateral de nódulo em região submandibular. B: visão lateral da massa em região submandibular esquerda sendo mensurada sua maior medida $(8,5 \mathrm{~cm}$ de comprimento) com auxílio de um paquímetro.

Foram solicitados exames de imagem de radiografia torácica, em três projeções, e ultrassonografia abdominal, não havendo alterações dignas de nota. A fim de verificar a possibilidade cirúrgica foi 
solicitado exame de ultrassonografia cervical, sendo descrita lesão expansiva de margens pouco definidas e superfície irregular, heterogênea, de ecogenicidade mista, com áreas cavitárias de conteúdo anecogênico. Foi observado ainda a compressão da veia jugular, porém, sem acometimento luminal. Os linfonodos cervicais se apresentavam aumentados. Foi verificada a possibilidade de excisão cirúrgica, porém o tutor optou por realizar o procedimento em clínica veterinária externa.

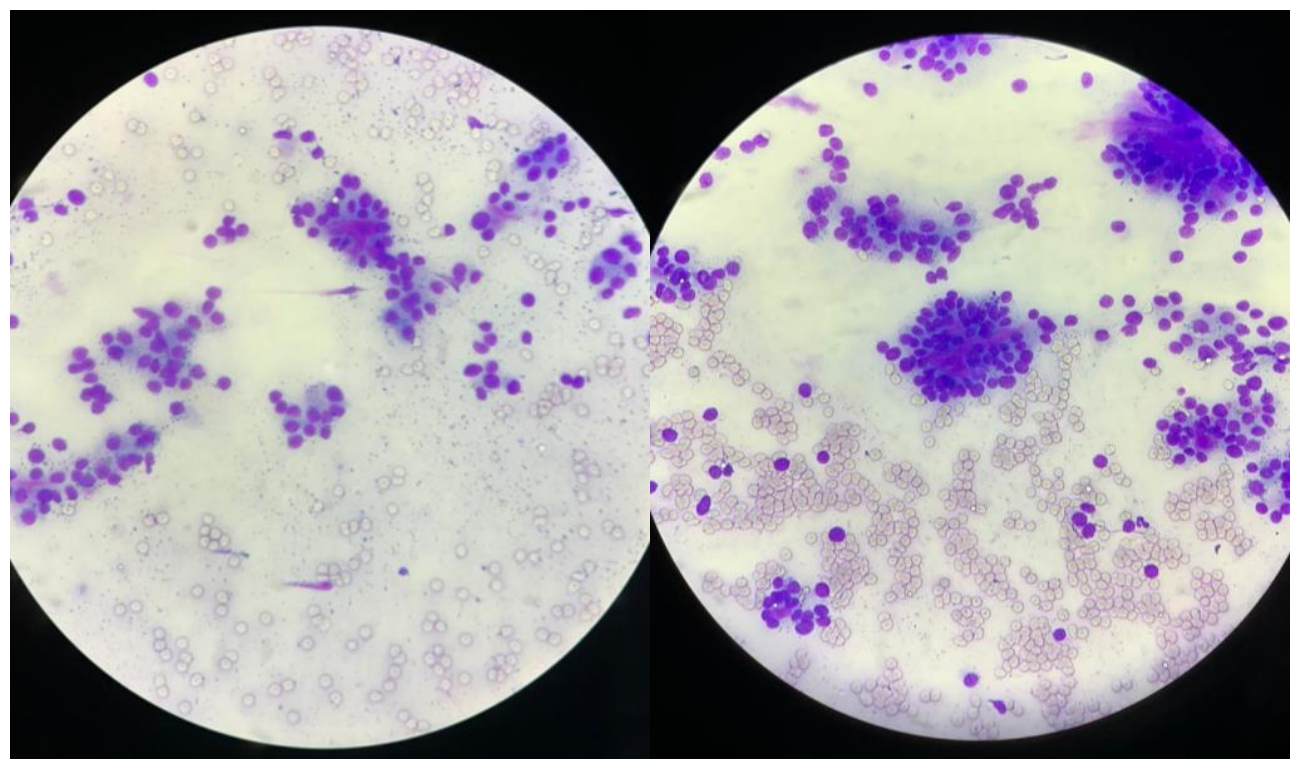

Figura 2. Citologia com resultado compatível com neoplasia epitelial glandular, com evidência de critérios de malignidade de anisocitose, anisocariose, relação núcleo: citoplasma aumentada, dentre outros.

O animal retornou ao serviço de oncologia após a excisão cirúrgica, sem informações acerca da margem empregada na excisão, sendo considerado que as margens se encontravam comprometidas. Foi indicada a radioterapia como primeira opção, sendo recusada pelos tutores por questões financeiras. Diante do quadro, foi recomendado tratamento quimioterápico convencional paliativo em protocolo utilizando a combinação da doxorrubicina na dose de $30 \mathrm{mg} / \mathrm{m}^{2}$ administrada no dia 1 ; e da ciclofosfamida na dose de $50 \mathrm{mg} / \mathrm{m}^{2}$ administrada nos dias 2, 3, 4 e 5; totalizando 4 ciclos com intervalos de 21 dias. O animal permaneceu em tratamento até a quarta sessão, porém veio a óbito na semana seguinte, manifestando sinais de recidiva tumoral e acometimento linfonodal. A sobrevida total constatada foi de 120 dias a partir do diagnóstico inicial por biopsia excisional.

\section{Discussão}

As neoplasias de glândula salivar são consideradas raras, com poucos dados epidemiológicos comparativos disponíveis em literatura, nacional e internacional. Em cães não há predileção por raça ou sexo (Meuten, 2016); porém, Hammer et al. (2001) afirmam em um estudo retrospectivo uma predileção para machos felinos, sem comprovação em cães. Em relação à idade, sugere-se que os animais idosos são mais frequentemente afetados (Carberry, 1988; Meuten, 2016; Sánchez et al., 2005). No presente relato, o animal apresentava idade avançada, sendo a faixa etária mais acometida pela maioria dos processos neoplásicos, incluindo as neoplasias de glândula salivar.

Apesar de alguns casos reportados serem classificados como assintomáticos, a presença da massa com aumento de volume local é um achado frequente (Hammer et al., 2001). Também é citado halitose, perda de peso, anorexia, disfagia, exoftalmia, síndrome de Horner, espirros e disfonia como sintomatologia observada (Hammer et al., 2001; Meuten, 2016; Withrow et al., 2020). O animal se manifestou assintomático mesmo após o agravamento da doença, apresentando unicamente o aumento de volume local recorrente após a excisão.

Para o diagnóstico, o exame citológico pode ser sugestivo, auxiliando na diferenciação dos tumores de glândula salivar em relação aos demais tecidos e lesões de localização cervical (Militerno et al., 2005). Todavia, o exame histopatológico é necessário para confirmar o diagnóstico (Liptak \& Withrow, 2007). A realização de ambos os exames, com resultados concordantes, conforme realizado no caso 
descrito fornece um diagnóstico mais confiável e preciso. Ambos os procedimentos foram realizados em clínicas externas e não contaram com a avaliação de margem cirúrgica.

Exames de imagem como ultrassonografia cervical e tomografia computadorizada cervical e de cabeça podem auxiliar no diagnóstico, além de contribuir na decisão e planejamento terapêutico (Withrow et al., 2020). A ultrassonografia cervical mostra vantagens com alta precisão diagnóstica, baixa invasividade, ausência de exposição à radiação, alta reprodutibilidade e baixo custo (Welkoborsky, 2011) e se combinado com a imagem Doppler colorida, permite a avaliação da vascularização sugerindo a natureza da lesão (Rastogi et al., 2012). Já a TC fornece maior precisão de informações (Lenoci \& Ricciardi, 2015). No presente relato foi indicado exame de tomografia computadorizada, porém, os tutores não realizaram por restrições financeiras, sendo optado pela ultrassonografia cervical. $\mathrm{O}$ exame se mostrou determinante no manejo do caso.

Como protocolo terapêutico é indicada a completa ressecção cirúrgica, respeitando as margens de segurança preconizadas (Hammer et al., 2001), com a quimioterapia indicada com fins paliativos (Evans \& Thrall, 1983). Não existe um protocolo padrão de quimioterapia estabelecido para esse tipo neoplásico; porém, cita-se a utilização de diversos agentes como a carboplatina, cisplatina, ciclofosfamida, clorambucil, doxorrubicina, mitoxantrona, vincristina, entre outros (Hammer et al., 2001). No presente relato empregou-se a quimioterapia de forma adjuvante e paliativa, após a ressecção cirúrgica, diante da impossibilidade de execução da radioterapia. A associação da doxorrubicina e ciclofosfamida, conforme utilizada no presente trabalho, é amplamente utilizada, com indicação no tratamento de outros tumores de origem glandular como os carcinomas mamários e de tireoide (Daleck et al., 2016), havendo relatos dessa combinação também na medicina humana, no tratamento dos carcinomas de glândula salivar (Dreyfuss et al., 1987).

Considera-se que o diagnóstico precoce melhore significativamente o tempo de sobrevida (Hammer et al., 2001; Sidaway et al., 2004), e que as chances de cura são maiores sempre na primeira intervenção terapêutica. No entanto, outros fatores prognósticos ainda são desconhecidos para as neoplasias de glândula salivar (Withrow et al., 2020). No presente relato, apesar do diagnóstico precoce, o animal foi submetido a dois procedimentos cirúrgicos sem a obtenção de margens livres, fator que pode contribuir para o comprometimento do sucesso terapêutico. A sobrevida atingida permaneceu em 120 dias, inferior ao relatado por Almeida et al. (2010) que observaram uma sobrevida de 180 dias com protocolo quimioterápico à base de carboplatina pós-remoção cirúrgica.

\section{Conclusão}

Em casos de aumento de volume submandibular o clínico deve sempre considerar a possibilidade de neoplasia, mesmo com a baixa incidência dos tumores de glândula salivar. Essa neoplasia ainda representa um desafio, relacionado principalmente ao seu grau de malignidade, agressividade, e a escassez de estudos relacionados. Dessa forma, mais estudos são necessários principalmente em relação à terapêutica aplicada e aos protocolos quimioterápicos.

\section{Referências}

Almeida, A. P., Malm, C., Lavalle, G., Cassali, G. D., Santos, R. L., \& Paixão, T. A. (2010). Salivary gland carcinosarcoma in a dog. Brazilian Journal of Veterinary Pathology, 3(2), 137-141. DOI: https://doi.org/10.1016/j.jcpa.2012.11.089

Carberry, C. (1988). Salivary gland tumors in dogs and cats: a literature and case review. Journal American Veterinary Medical Association, 24, 561-567.

Collins, T., Cotran, R. S., \& Kumar, V. (2000). Patologia estrutural e funcional. Guanabara, Koogan.

Daleck, C. R., Fonseca, C. S., \& Canola, J. C. (2016). Oncologia em cães e gatos. Roca.

Dreyfuss, A. I., Clark, J. R., Fallon, B. G., Posner, M. R., Norris Jr, C. M., \& Miller, D. (1987). Cyclophosphamide, doxorubicin, and cisplatin combination chemotherapy for advanced carcinomas of salivary gland origin. Cancer, 60(12), 2869-2872. DOI: https://doi.org/10.1002/10970142(19871215)60:12<2869:aid-cncr2820601203>3.0.co;2-y

Evans, S. M., \& Thrall, D. E. (1983). Postoperative orthovoltage radiation therapy of parotid salivary 
gland adenocarcinoma in three dogs. Journal of the American Veterinary Medical Association, 182(9), 993.

Hammer, A., Getzy, D., Ogilvie, G., Upton, M., Klausner, J., \& Kisseberth, W. C. (2001). Salivary gland neoplasia in the dog and cat: survival times and prognostic factors. Journal of the American Animal Hospital Association, 37(5), 478-482. DOI: https://doi.org/10.5326/15473317-37-5-478

Head, K. W. (2003). Histological classification of salivary gland tumors of domestic animals. The Armed Forces Institute of Pathology.

Lenoci, D., \& Ricciardi, M. (2015). Ultrasound and multidetector computed tomography of mandibular salivary gland adenocarcinoma in two dogs. Open Veterinary Journal, 5(2), 173-178.

Liptak, J. M., \& Withrow, S. J. (2007). Cancer of the gastrointestinal tract. In S J Withrow \& D. M. Wail (Eds.), Small animal clinical oncology. (pp. 455-473). Saunders Elsevier.

Meuten, D. J. (2016). Tumors in domestic animals. John Wiley \& Sons.

Militerno, G., Bazzo, R., \& Marcato, P. S. (2005). Cytological diagnosis of mandibular salivary gland adenocarcinoma in a dog. Journal of Veterinary Medicine Series A, 52(10), 514-516. DOI: https://doi.org/10.1111/j.1439-0442.2005.00767.x

Rastogi, R., Bhargava, S., Mallarajapatna, G. J., \& Singh, S. K. (2012). Pictorial essay: Salivary gland imaging. The Indian Journal of Radiology \& Imaging, 22(4), 325-333.

Rosenthal, R. C. (2004). Segredos em oncologia veterinária. Artmed.

Sánchez, J., Buendía, A. J., Vilafranca, M., Velarde, R., Altimara, J., Martínez, C. M., \& Navarro, J. A. (2005). Canine carcinosarcomas in the head. Veterinary Pathology, 42(6), 828-833.

Sidaway, B. K., McLaughlin, R. M., \& Hughes, J. (2004). Cervical masses in dogs: diagnosis and treatment. Compendium on Continuing Education for the Practicing Veterinarian, 5, 390-402.

Sozmen, M., Brown, P. J., \& Eveson, J. W. (2003). Salivary gland basal cell adenocarcinoma: a report of cases in a cat and two dogs. Journal of Veterinary Medicine Series A, 50(8), 399-401. DOI: https://doi.org/10.1046/j.0931-184x.2003.00563.x

Spangler, W. L., \& Culbertson, M. R. (1991). Salivary gland disease in dogs and cats: 245 cases (19851988). Journal of the American Veterinary Medical Association, 198(3), 465-469.

Welkoborsky, H. J. (2011). Current aspects in ultrasonography of the salivary glands. HNO, 59(2), 155165.

Withrow, Stephen J, Page, R., \& Vail, D. M. (2020). Small animal clinical oncology. Elsevier Health Sciences.

\section{Histórico do artigo:}

Recebido: 8 de julho, 2020.

Aprovado: 6 de agosto, 2020.

Disponível online: 23 de novembro, 2020.
Licenciamento: Este artigo é publicado na modalidade Acesso Aberto sob a licença Creative Commons Atribuição 4.0 (CC-BY 4.0), a qual permite uso irrestrito, distribuição, reprodução em qualquer meio, desde que o autor e a fonte sejam devidamente creditados 